\title{
Socionomics: The Way Business Transforms with Social Media
}

\author{
K. Sasi Kala Rani ${ }^{1}$, U. Susmitha ${ }^{1}$, D. Ramya ${ }^{1}$ and K. Sujatha ${ }^{1,2}$ \\ 'Department of Computer Science, Sri Krishna College of Engineering and Technology, Coimbatore - 641008, Tamil \\ Nadu, India; sasikalaranik@skcet.ac.in,17epcs018@skcet.ac.in, ramya2d@gmail.com,sujatha.ssps@gmail.com \\ Wenzhou Kean University, China.
}

\begin{abstract}
Objectives: To develop an enhanced web application, using web services for interconnecting social network, E-Commerce application and News channels. Products will be recommended based on customers' preferences. Methods/Statistical Analysis: A three-tier methodology in which social network is the decision-making layer and E-commerce and news channel portals are the application layers. The social media layer will be analyzed using a method called as Text categorization which generates data for gathering user preferences. This information will be made as micro-blogging information. This will be passed on to other networks via Artificial Neural Network (ANN). Findings: The micro-blogging details are created at the tier one, Social network. This is then connected to e-commerce website and news portals. The micro-blogging information created will be purely based on users' preferences, hobbies and other user data provided in the social network. These are further passed on to the e-commerce website to accurately recommend the products to individual users. Also, on the news portal, individual users can get notified about local news about their localities. Application/Improvements: This serves as a three-in-one application since there are three different tiers. Further improvements could be made by adding offline features. This could be improved by developing this as a separate mobile application.
\end{abstract}

Keywords: E-Commerce, Recommender System, Social Media, ANN

\section{Introduction}

Nowadays, social media spending makes up a small fraction of most business marketing budgets. A recent Duke University survey found that, on an average, social media spending accounted for just $9 \%$ of the overall budget. In any case, that number is anticipated to grow to about $22 \%$ in the following five years. Plainly, internet business advertisers perceive the intensity of internetbased life to interface with a group of people. Facebook, Twitter, LinkedIn, and Instant gram are nearly ubiquitous in our lives. They're similar to the 21st-Century Main Street; we use them to impart, discover data rapidly, and progressively, to look for items. For Web organizations, powerful social promoting speaks to genuine esteem. Interpersonal organizations offer better approaches to achieve first-time clients, lock in also, remunerate existing clients, and exhibit the best your image brings to the table.
The social network profiles and the contents shared are very important $\frac{1,2}{}$.

Organizations that incorporate web-based life into their advertising procedure - from client procurement, to deals, to re-commitment crusades - will have advantage. Advertisers can find continuously what a group of onlookers thinks about most, their interests, the discussions they have and their likings. Utilizing such information may help in the optimization of campaigns and deliver more targeted messaging. Web-based social networking makes it simpler to spot and react to horrendous client encounters. Building up a methodology for reacting to client request by means of online life becomes the need of the hour.

Electronic commerce continues to grow in popularity around the world. In 2007, more than $85 \%$ of Internet users worldwide had made an online purchase. In the United States, by 2014, $8 \%$ of retail sales will be made through e-commerce and more than half of all retail 
sales will be made online or will be affected by online research. Social networking sites are exploding in growth. $72 \%$ of Internet users in the world are active on a social networking site. Although blocked in China, Facebook is now the most popular social networking site in the world with more than 583 million members ${ }^{3,4}$.

\section{Existing Methodologies}

\subsection{Amazon.com Recommendations: Item-to-Item Collaborative Filtering}

Amazon.com utilizes proposal calculations to customize the on the web store for every client. The store profoundly changes dependent on client interests, appearing programming titles to a product architect and child toys to another mother. Online business suggestion calculations frequently work in a testing situation. For example:

A large retailer can have huge amounts of data, tens of millions of customers and millions of different catalog items. Numerous applications require that the result set be returned progressively, in almost a large part of a second, while still generating fantastic suggestions. New clients regularly have amazingly restricted data, in view of just a couple of buys or item evaluations. More seasoned clients can have a overabundance of data, in light of thousands of buys and evaluations. Client information is unpredictable: Each association provides important information to the client, and the calculation must react quickly to the new data. There are three basic ways to deal with the suggestion problem: regular synergistic screening, group models and research-based techniques. Here, we compare these methods with our algorithm, which we call collaborative filtering from element to element. Amazon.com uses the recommendations as a targeted marketing tool in many email campaigns and on most pages of their websites, including Amazon's high traffic.com home page. By clicking on "Your recommendations", connect customers to a region where they can channel their proposals by product offer and branch of knowledge, rate prescribed

\section{Your Recommendations}

Software Requirements

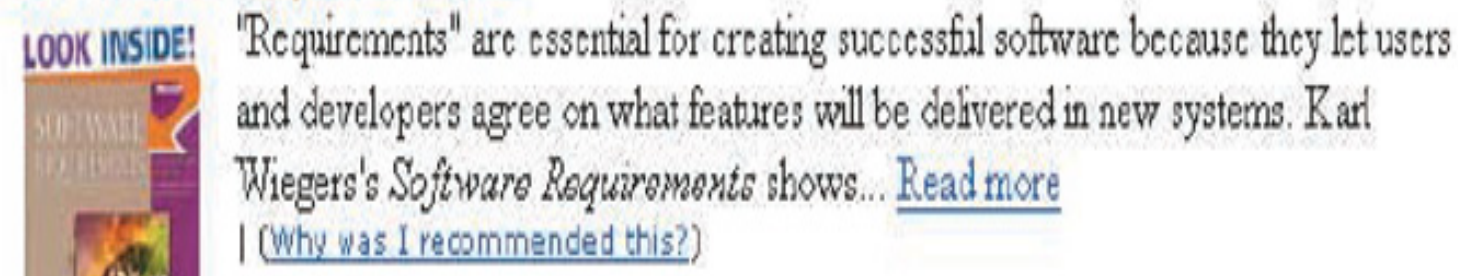

More Recommendations

Star Wars - Episode L, The Phantom Menace DVD Liam Neeson (Why?)

The Sopranos - The Complete Second Season DVD) Sopratios (whu?)

Doaih March by Edward Yourdon (why?)

The Pragmatic Programmer by Andrew Hunt, et al (Why?)

Figure 1. The "Your Recommendations" features on Amazon.com homepage. Using this feature, customers can sort recommendations and add their own product ratings.. 


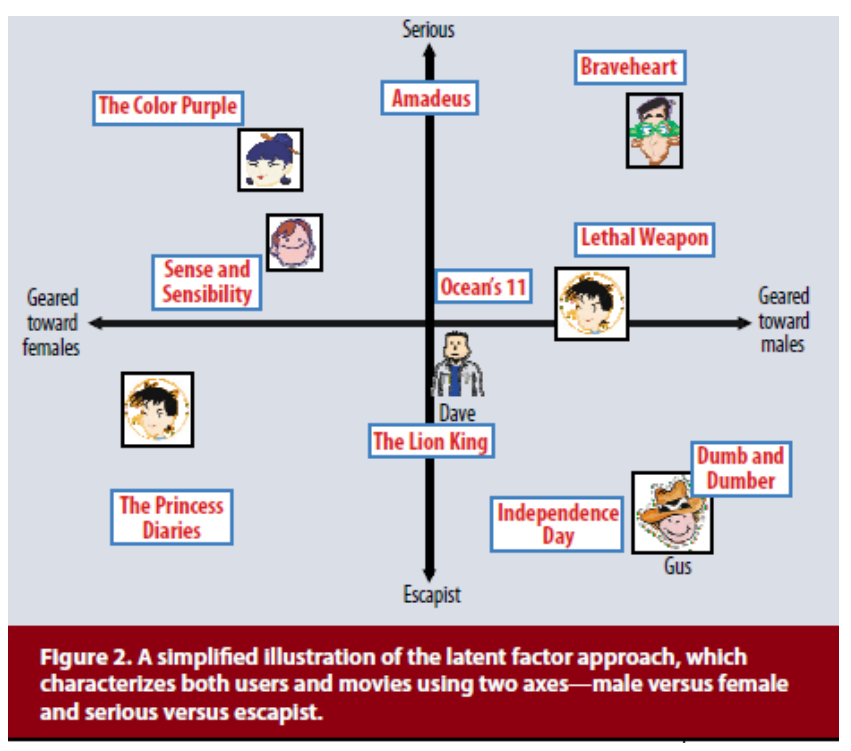

Figure 2. Latent factor approach-finds connections between users and objects to get new associations..

items, rate their previous purchases and see why things are suggested as shown in Figure 1 and 2, our shopping cart recommendations, which offer customers suggestions for products based on the items in their shopping cart. The component is like the motivational things in the line of exit of a store of groceries; nevertheless, our things of unit are focused to each client. Amazon.com makes extensive use of suggestion calculations to customize its website to the advantages of each client $\underline{5}$.

\section{Advantages}

- Unlike traditional collaborative filtering, online computation scales independent of the number of customers and number of items in the product catalogue.

- Produces recommendations in real-time.

- Can be used for massive data sets.

- Generates high quality recommendations.

\section{Disadvantages}

- Cold start problem: inability to address the system's new products and users.

\subsection{Matrix Factorization Techniques for Recommender Systems}

Modern consumers are in undated with options. Electronic retailers and substance suppliers offer an immense selection of items, with phenomenal possibilities to satisfy a variety of unusual needs and tastes. Coordinating buyers with the most appropriate items is essential to improve customer compliance and reliability. Therefore, more retailers have shown interest in the recommendation systems, which examine examples of the customers' enthusiasm for the articles to offer personalized proposals that adapt to the tastes of the customers. Since great personalized suggestions can add another measure to the client's encounter, web-based business pioneers like Amazon.com and Netflix have made recommendation systems a surprising piece of their sites. An alternative to content filtering is based solely on the behavior of the previous user, for example, previous transactions or product ratings, without requiring the creation of explicit profiles. This approach is known as collaborative filtering. Collaborative filtering analyzes the relationships between users and the interdependencies between products to identify new associations of user elements. The two essential territories of community selection are area strategies and inactivity factor models. Neighborhood methods focus on calculating the relationships between elements or, alternatively, between users. The article-oriented methodology evaluates a client's inclination for something that depends on evaluations of "neighboring" things by a similar client. The neighbors of an element are different elements that, in general, will obtain comparative evaluations when they are evaluated by a similar client ${ }^{\underline{6}}$.

\section{Advantages}

- Create a profile for each user or product to characterize nature. Profiles allow projects to connect clients with coordination elements.

- Collaborative filtering is domain-free and more accurate than content-based filtering.

\section{Disadvantages}

- Requires gathering external info that may not be accurate or easily available

- Suffers from the cold start/user problem-due to its inability to address the system's new products and user.

\subsection{A Social Network-based Recommender System (SNRS)}

The social impact assumes a critical job in the promotion of articles. In any case, it has rarely been considered in conventional recommendation systems. This document presents a new paradigm of recommendation systems that can use information in social networks, including user preferences, general acceptance of the article and the influence of social friends. A probabilistic model is created to make personalized proposals based on said data. The data is extracted from a real online social network, and the analysis of this large data set reveals that friends have 
a tendency to select the same elements and give similar ratings as shown in Figure 3.

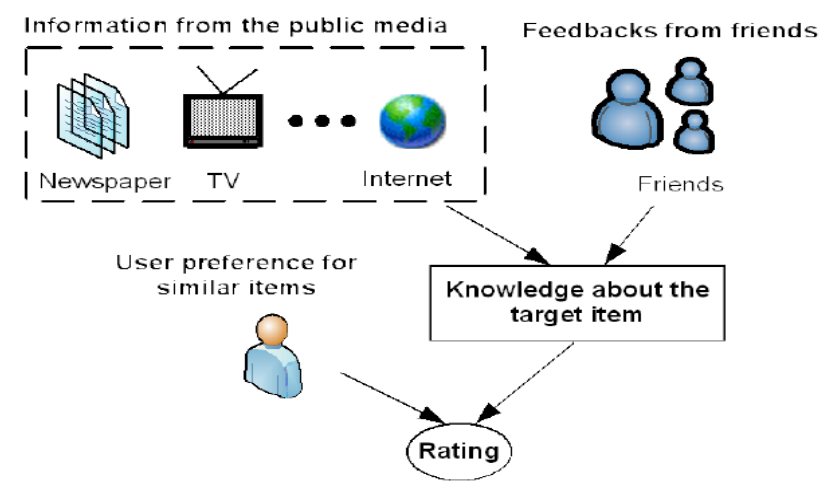

Figure 3. The three factors that influence a customer's buying decision: user preference for similar items, information regarding the target item from the public media, and feedbacks fromfriends.com.

Intuitively, when someone wants to buy a product that is not familiar, often consult with friends who already have experience with the product. When friends recommend a product, we also tend to accept such a recommendation because the tickets are reliable. Many marketing strategies that have taken advantage of this aspect of human nature and have achieved great success. A classic example is Hotmail's free email service. The marketing strategy of Hotmail is to attach a promotional message at the bottom of each outgoing email: "Get your private and free email at http://www.hotmail.com". The people who receive the email will join and spread more by forwarding the message. As a result, the number of Hotmail user accounts increased from zero to 12 million in 18 months with an advertising budget of only $\$ 500,000$, which outperformed many conventional marketing strategies. Therefore, social impacts assume a key role when individuals are deciding the options of receiving articles ${ }^{7}$.

\section{Advantage}

- Improves prediction accuracy by $17.8 \%$

Disadvantage

- Lack of trusted users

\subsection{Product Recommendation and Rating Prediction based on Multi-Modal Social Networks}

Mainly there are two types of social networks: explicit and implicit social networks. Explicit social networks include adding each other as friends and implicit social networks include commenting/rating a product. This article proves how the performance of Social-Union, CF based on the user and Friend TNS is affected, when we apply different controllable densities in the friendship network and in the network of classification of user elements, respectively.

Here, the performance of Social-Union is studied and, at the same time, the fraction of qualified elements and the edges observed in the user element and friendship networks respectively are increased.

\section{Methodology Used}

The methodology/algorithm used here is called the social union algorithm that combines multiple similarity matrices derived from explicit or implicit heterogeneous social networks. The Social Union exploits the characteristics of friendship networks and user elements, also exploiting the local characteristics of the graphics.

The paper proposes Social Union, a method that combines multiple similarity matrices derived from explicit or implicit heterogeneous social networks. Social-Union takes into account the local and global characteristics of the graphics, such as the density of the graphics, the density of the user profile, the structure of the nodes, etc. In addition, they present a well-defined framework for combining heterogeneous social networks ${ }^{8}$.

\section{Advantages}

- One of the main merits is that it has more accurate item recommendation mechanism.

\section{Disadvantages}

- The disadvantage is that a friend recommendation mechanism is absent.

\section{Proposed Methodology}

In the proposed system we are using a 3-tier architecture. The three networks are interconnected with other networks. The architecture has been divided into layers. The social network is the decision-making layer and other two layers are the application layers.

The social network will be analyzed using text categorization methods which will deal with all the personal information like education, location, hobbies, comments, posts etc. The data from the social networks will be taken into consideration for gathering information from the other networks. In case the user may change the profile details, then the user's recommendation also changes. This process is done automatically. The gathered information will be created as the micro blogging 
information. This information is the alpha numerical secret words which will be passed on from one network to another using artificial neural network (ANN). The ANN acts as the bridge between these systems for efficient data transfer and retrieval. This connects both commerce and news channels. The news channels contain more text information which can be accessed by text categorization.

And finally, the data will be published in the head network i.e., the social network. A panel will be designed in the front end for displaying all the recommended information. All the displayed information is more relevant to the user profile and user need.

\section{Advantages of the Proposed System}

- Three tier architecture has been implemented, so that each network will be interconnected with the other network, which is called as a hybrid network.

- The special enhancement has been done for the micro blogging information. All the information will be converted into alpha numerical characters.

- All information will be taken into consideration, like personal info, education details, location details, posts and comments.

- More security has been established; in case the failure of any network, other network will work perfectly.

A product application when all is said in done

is executed subsequent to exploring the total life cycle strategy for an undertaking. Different life cycle procedures, for example, prerequisite investigation, structure stage, confirmation, testing lastly pursued by the execution stage results in an effective venture the board a product application by and large is actualized in the wake of exploring the total life cycle strategy for a venture. Different life cycle procedures, for example, necessity examination, structure stage, check, testing lastly pursued by the usage stage results in an effective task the executives ${ }^{9,10}$ as shown in Figure 4.

\section{Findings}

Table 1, gives the performance information of various tiers of the application. The proposed system's performance is quite high since it ensures the working of each tier independently.

\section{Conclusion}

The micro blog creates an internal data transfer for efficient data retrieval from the three networks. Displaying name and news will be change according to the user profile information. All the displaying news and products are more relevant to the users. As mentioned in the abstract, now the $89 \%$ of the internet users may use

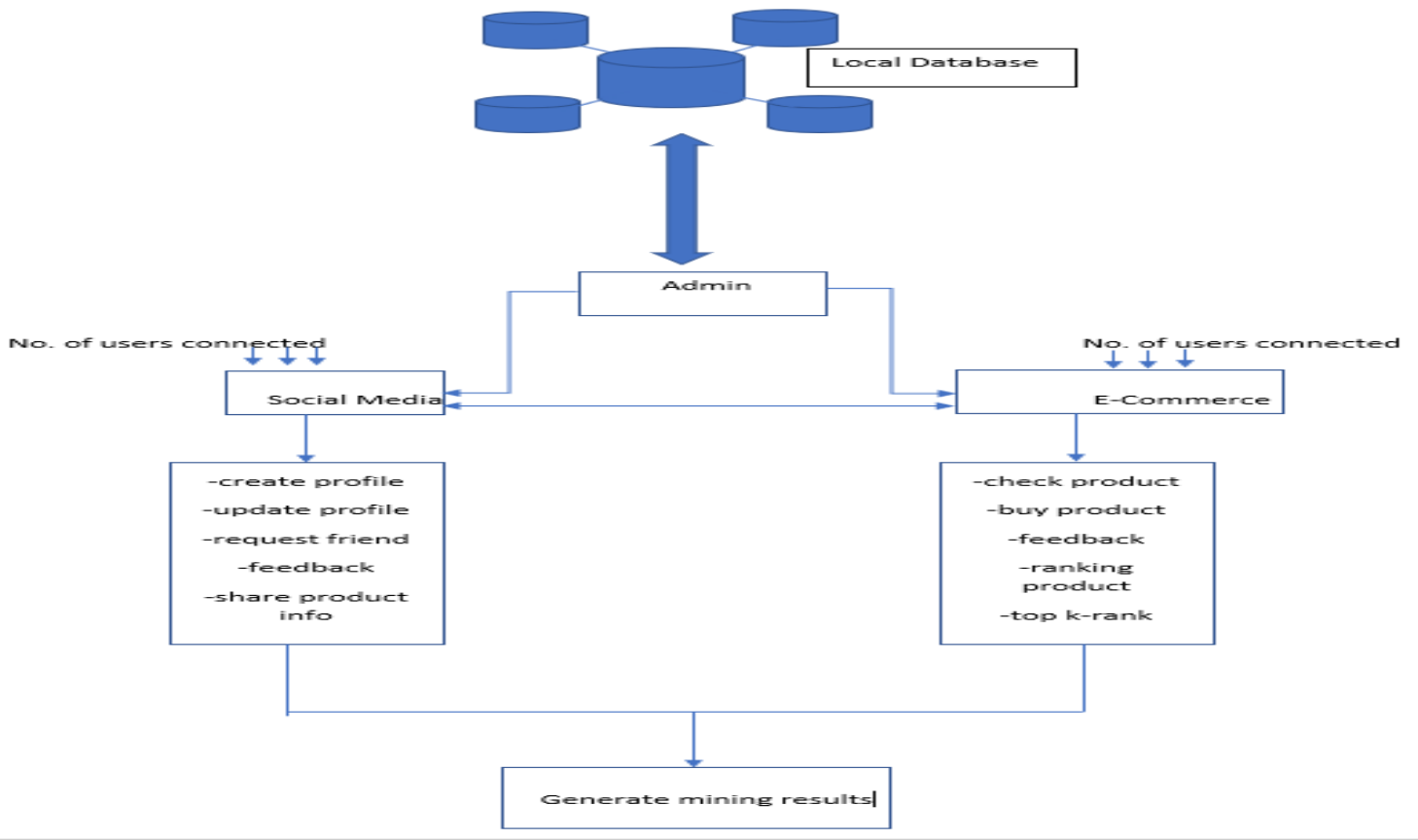

Figure 4. System architecture - an idea on the logical design of the system which consists of the Admin, the tiers and the results. 
Table 1. Performance comparison between existing and proposed systems

\begin{tabular}{|l|l|l|l|l|}
\hline \multicolumn{1}{|c|}{ Networks } & \multicolumn{1}{|c|}{ Independent performance } & Existing performance & $\begin{array}{l}\text { Current performance } \\
\text { (Proposed system) }\end{array}$ & \multicolumn{1}{c|}{ Micro blog Ratio } \\
\hline Social Networks & $\begin{array}{l}\text { Internet traffic and number of } \\
\text { users. Supports up to 5 M users }\end{array}$ & $\begin{array}{l}\text { Depends on server and } \\
\text { internet speed }\end{array}$ & $\begin{array}{l}\text { Tier 1 architecture. 93\% } \\
\text { works independently }\end{array}$ & $\begin{array}{l}\text { Creates Micro blogging } \\
\text { and receives contents }\end{array}$ \\
\hline $\begin{array}{l}\text { E-commerce } \\
\text { application }\end{array}$ & $\begin{array}{l}\text { Contains payment gateways. } \\
\text { Slow performance during } \\
\text { money transactions }\end{array}$ & $\begin{array}{l}\text { Depends on server and } \\
\text { internet speed }\end{array}$ & $\begin{array}{l}\text { Tier } 2 \text { architecture } 40 \\
\% \text { depends on social } \\
\text { network }\end{array}$ & $\begin{array}{l}\text { Receives Micro blogging } \\
\text { and transfer contents to } \\
\text { tier } 1\end{array}$ \\
\hline News channels & $\begin{array}{l}\text { Contains sensitive information. } \\
\text { Need networks to spread news }\end{array}$ & $\begin{array}{l}\text { Depends on server and } \\
\text { internet speed }\end{array}$ & $\begin{array}{l}\text { Tier 3 architecture 60 } \\
\% \text { depends on social } \\
\text { networks }\end{array}$ & $\begin{array}{l}\text { Receives Micro blogging } \\
\text { and transfer contents to } \\
\text { tier } 1\end{array}$ \\
\hline
\end{tabular}

the $60 \%$ of the ecommerce application. This makes more sales in the ecommerce application. And also according the news concept, $89 \%$ of the social network users will view all the news. This makes all the network users raised to $89 \%$ among the internet users. More information can be viewed in a single screen in less mobile data.

\section{Future Enhancements Possible are:}

- Layers can be users instead of three tier architecture.

- Layers need live internet buffer data and API key from Google. These are expensive, but by implementing layers concept in this application, live traffic updates and weather updates can be given.

- Mobile responsive web application.

- A dedicated mobile application can be developed using Android, IOS, Blackberry and Windows. So that people can enjoy the responsive mobile based web application.

- Offline features

- By using adaptive memory management, offline features can be implemented. This feature will load most of the information during online and access or download the data during the offline mode.

\section{References}

1. Wang J, Yi Z. Opportunity model for e-commerce recommendation: right product; right time. Proceedings of the 36th International ACM SIGIR Conference on Research and Development in Information Retrieval. 2013; p. 303-12. https://doi.org/10.1145/2484028.2484067

2. GieringM.Retailsalespredictionanditem recommendations using customer demographics at store level. ACM SIGKDD
Explorations Newsletter. 2008; 10(2):84-9. https://doi. org/10.1145/1540276.1540301

3. Linden G, Smith B, York J. Amazon.Com recommendations: Item-to-item collaborative filtering. IEEE Internet Computing. 2003; 1:76-80. https://doi.org/10.1109/ MIC.2003.1167344

4. Zhao XW. We know what you want to buy: a demographicbased system for product recommendation on microblogs. Proceedings of the 20th ACM SIGKDD International Conference on Knowledge Discovery and Data mining. 2014; p. 1-10. https://doi.org/10.1145/2623330.2623351

5. Wang J. Leveraging Product Adopter Information from Online Reviews for Product Recommendation. International Conference on Web and Social Media. 2015; p. 1-9.

6. Seroussi Y, Fabian B, Ingrid Z. Personalized rating prediction for new users using latent factor models. Proceedings of the 22nd ACM Conference on Hypertext and Hypermedia. 2011; p. 47-56. https://doi.org/10.1145/1995966.1995976

7. Lin J. Addressing cold-start in app recommendation: latent user models constructed from twitter followers. Proceedings of the 36th International ACM SIGIR Conference on Research and Development in Information Retrieval. 2013; p. 1-10. https://doi.org/10.1145/2484028.2484035

8. Koren Y, Robert B, Chris V. Matrix factorization techniques for recommender systems. Computer. 2009; 8:30-7. https:// doi.org/10.1109/MC.2009.263

9. Rendle S. Social network and click-through prediction with factorization machines. KDD-Cup Workshop. 2012; p. 1-38.

10. Zhang M. Addressing cold start in recommender systems: A semi-supervised co-training algorithm. Proceedings of the 37th International ACM SIGIR Conference on Research \& Development in Information Retrieval. 2014; p. 1-10. https://doi.org/10.1145/2600428.2609599 